\title{
Index rerum ad Vol. 14
}

ABO groups and serum alkaline phos-

phatases 289

Analgesia, congenital 133

clinico-genetical studies 143

- $\quad$ cytogenetic studies 133

Animal (pig) blood groups 319

Australia, dermatoglyphics 201

Beta-globulins, abnormal, in Hawaiians

314 Birth order and sex ratio 177 Blood groups and serum alkaline phos-

phatases 286

in Leber's optic atrophy 234

in pigs 319

see also ABO and N2

Brazilian Indians, colour blindness 212 Canada, fertility rates 186 Cancer and diabetes mellitus

116 Cardiovascular lesions and diabetes mellitus 116 Colour blindness in Brazilian Indians

212 Congenital analgesia, see Analgesia, congenital Congenital malformations, classification

system 76 Cytogenetic studies in congenital analgesia 133 Cytogenetics, see also Down's

syndrome Dermatoglyphic pattern variability 201 Diabetes mellitus, inheritance 97 interrelationship with other diseases

113 Down's syndrome, chromosomal analyses in families with multiple occurrence 251 seasonal birth variations 269

triple chromosomal mosaicism 67

Dwarfism with bone dysplasia of extremities 63 Fertility and mother's age 186

- $\quad$ rates (Canada) 186

Finger prints, see Dermatoglyphics Gene frequency variations in panmictic

populations 351 Germany (South-Western), Gm and Inv

serum groups $298 \mathrm{Gm}$ serum groups, formal genetics 4, 96

in Hawaii 25

in South Western Germany 298

$\mathrm{H}$ blood group system in pigs 319

Haptoglobins in Hawaiians 311

Northern Italy 36

- $\quad$ Variations in newborn children 159

Hawaiian population groups, serum pro

tein variations 309

Incompatibility between mothers and

male foetuses 177 Indians, Brazilian, colour blindness 212 Indian tribes, PTC sensitivity 168 Inv serum groups, formal genetics 15

in Hawaii 25 
in South Western Germany 298

Italy (Northern), Hp groups 36

Leber's optic atrophy with neurological

symptoms 220 Lewis groups and serum alkaline phosphates 290 Linkage, practical possibilities of detection 125 Lyon's hypothesis and dermatoglyphic

patterns 207 Malformations, congenital, see Congenital

malformations Mental deficiency and season of birth 265 MN blood groups, see N2 antigen Mongolism, see Down's syndrome N2 antigen variations 57 Newborns, haptoglobin variations

159 Nystagmus, hereditary 240 Optic atrophy, see Leber Phenylketonuria, detection of heterozygotes 327 Population genetics, colour blindness in Brazilian Indians 212

dermatoglyphics in Australia 201

fertility rates in Canada 186

Gm groups in Hawaii 25

South Western Germany 298

Haptoglobins in Hawaii 212

Northern Italy 311

- $\quad$ Inv groups in Hawaii 25

South Western Germany 298

PTC sensitivity in some indian tribes

168

Transferrin variants in Italy 36

world distribution 41

Phosphatases, alkaline, and blood groups

286

Index rerum

375

Pseudocholinesterase variants, fluoride resistant type 281

nomenclature 366

PTC taste sensitivity in some Indian tribes 168

Secretor character and serum alkaline phosphatases 290

Serum groups, see also Alkaline phosphatases, Gm groups, Inv groups, Pseudocholinesterase and Transferrins

Sex differences in dermatoglyphic patterns

203

Sex ratio in humans 177

Tooth eruption phases 338

Transferrin D variants 41, 52

- in Hawaiians 313

Northern Italy 36 